\title{
Potencial de sistemas agroflorestais multiestrata para sequestro de carbono em áreas de ocorrência de Floresta Atlântica
}

\author{
Luís Cláudio Maranhão Froufe ${ }^{1}$, Marcos Fernando Gluck Rachwal ${ }^{1}$, Carlos Eduardo Sicoli Seoane ${ }^{1}$ \\ ${ }^{1}$ Embrapa Florestas, Estrada da Ribeira, Km 111, CP 319, CEP 83411-000, Colombo, PR, Brasil
}

"Autor correspondente:

luisão@cnpf.embrapa.br

Termos para indexação:

Conservação dos recursos naturais

Floresta Ombrófila Densa

Estoque de carbono

SAF

Sistemas de uso do solo

Index terms:

Agroforestry systems

Atlantic Rain Forest

Carbon sink

Land use

Natural resource conservation

Histórico do artigo:

Recebido em 22 nov 2010

Aprovado em 13 jun 2011

Publicado em 30 jun 2011

doi: 10.4336/2011.pfb.31.66.143
Resumo - Foi avaliado o estoque de carbono no solo, serapilheira, biomassa arbórea e biomassa herbácea de SAFs multiestratos, em comparação a capoeiras em diferentes estágios de regeneração, sistemas agrícolas convencionais e pastagem, todos na região do Alto Vale do Ribeira, SP. Nos Neossolos e Cambissolos, com baixos teores de carbono orgânico e similaridade dos valores de densidade aparente, as capoeiras contribuíram com $115,78 \mathrm{Mg} \mathrm{ha}^{-1}$ de carbono total estocado, seguidas dos SAFs $\left(75,37 \mathrm{Mg} \mathrm{ha}^{-1}\right)$, das áreas agrícolas $\left(47,07 \mathrm{Mg} \mathrm{ha}^{-1}\right)$ e das pastagens $\left(36,01 \mathrm{Mg} \mathrm{ha}^{-1}\right)$. Apesar do grande potencial de sequestro de carbono dos SAFs, há necessidade de melhoria em suas práticas silviculturais.

\section{Carbon sink potential of multistrata agroforestry systems at Atlantic Rain Forest}

\begin{abstract}
Carbon storage of agroforestry systems, regenerated areas, conventional agriculture and pasture was evaluated at Alto Ribeira Valley region, São Paulo State, Brazil, in different compartments of Land-use systems (LUS). In soil, classified as Entisols and Inceptisols, we found similarities among all LUS, dued to their low contents of organic carbon, and similar values of bulk density. The total carbon stocked on land-use systems, greater amounts were determined on regenerated areas $(115.78 \mathrm{Mg}$ $\mathrm{ha}^{-1}$ ), followed by agroforestry systems $\left(75.38 \mathrm{Mg} \mathrm{ha}^{-1}\right)$, agriculture (47.07 $\left.\mathrm{Mg} \mathrm{ha}^{-1}\right)$, and pasture (36.01 $\mathrm{Mg} \mathrm{ha}^{-1}$ ). Despite their conservative characteristic, the silvicultural practices of multistrata agroforestry systems have to be improved for forest production and carbon sequestration.
\end{abstract}

\section{Introdução}

Dentre os gases causadores do efeito estufa, o dióxido de carbono $\left(\mathrm{CO}_{2}\right)$ de origem antrópica, responsável por até $80 \%$ do aquecimento global (YU, 2004), tem na biomassa vegetal e nos solos seus maiores potenciais de absorção nos ecossistemas terrestres. Segundo o Relatório Especial sobre Uso da Terra, Mudança do Uso do Solo e Floresta (Penman et al., 2000), houve, entre 1990 e 2000, aumento de 6,3 Gt ano ${ }^{-1}$ na emissão de carbono no mundo, com o sequestro de apenas $2,3 \mathrm{Gt} \mathrm{C}$ ano $^{-1}$ pelos oceanos e $0,7 \mathrm{Gt} \mathrm{C}$ ano $^{-1}$ pelos ecossistemas terrestres, resultando em incrementos anuais de 3,3 Gt C. Há que se destacar, também, que há apenas 20 anos o sequestro de carbono pelos ecossistemas terrestres era de 1,7 Gt C e essa redução foi decorrente, entre outros, da conversão de áreas florestais em agrícolas (YU, 2004).

A ocupação do território brasileiro foi acompanhada da intensa utilização de seus recursos naturais nos diversos ciclos econômicos, em detrimento dos 
estoques de madeira nativa, do ambiente edáfico e da conservação hídrica das microbacias. A presença de cobertura vegetal nativa, geralmente vista como um empecilho ao desenvolvimento agrícola, promoveu quadros alarmantes de desmatamento na Mata Atlântica, cujos remanescentes representam de $11,4 \%$ a $16 \%$ de sua área original, em sua maioria $(>80 \%)$ distribuídos em fragmentos menores que 50 ha, com forte efeito de borda e muito distantes entre si (Ribeiro et al., 2009). Diante desse quadro, práticas que aliem a produção de alimentos com a manutenção da capacidade produtiva do solo são necessárias para reduzir novos desmatamentos de ampliação da fronteira agrícola.

A conversão de ecossistemas tropicais naturais, como a Amazônia, em sistemas agrícolas pode representar a emissão de 1 a $9 \mathrm{Gt} \mathrm{C} \mathrm{ha-1}^{-1} \mathrm{ano}^{-1} \mathrm{e}$, neste ponto, sistemas agroflorestais se apresentam com elevado potencial para sequestro de carbono e a mitigação da emissão dos gases de efeito estufa (GEE) (Mutuo et al., 2005).

Os sistemas agroflorestais (SAFs) são sistemas de manejo em que a presença do componente arbóreo, a diversidade de espécies e a grande produção de biomassa favorecem a sustentabilidade ambiental, através da ciclagem de nutrientes no ambiente edáfico (Franke et al., 1998). Os SAF multiestrata (ME), caracterizados pela alta diversidade de espécies e a ocupação vertical de diversos estratos, são bastante difundidos na Indonésia (Suyanto et al., 2005), em Gana (Isaac et al., 2007) e na América Latina (Caja-Giron \& Sinclair, 2001; Staver et al., 2001; Angel-Pérez \& Mendoza, 2004; Holguín et al., 2007). No Brasil, SAF-ME são predominantemente empregados na Amazônia Ocidental (Lehman et al., 2001; Santos et al., 2002) e no sul da Bahia (Schroth et al., 2002). No Alto Vale do Ribeira, há pouco menos de 20 anos, vêm sendo conduzidas experiências com SAFs agroecológicos nos municípios de Barra do Turvo, SP, e Adrianópolis, PR, seguindo as idéias preconizadas por Götsch (1997).

Desta forma, este trabalho tem como objetivo principal determinar o estoque de carbono nos SAFs multiestrata, comparativamente a outros sistemas de uso da terra (SUT) adotados na região do Alto Vale do Ribeira, como ferramenta auxiliar de avaliação desse sistema de cultivo em programas de recuperação da Reserva Legal de pequenas propriedades rurais.

Pesq. flor. bras., Colombo, v. 31, n. 66, p. 143-154, abr./jun. 2011

\section{Material e métodos}

\section{Caracterização da área de estudo}

O presente trabalho foi desenvolvido na região do Alto Vale do Ribeira, SP, entre julho de 2007 e março de 2008. Esta região possui um remanescente da Floresta Ombrófila Densa Atlântica, apresentando árvores perenifoliadas, com brotos foliares sem proteção à seca, e ocupando uma área tropical úmida, sem período biologicamente seco (até 60 dias secos) durante o ano (Veloso \& Góes-Filho, 1982). O Município de Barra do Turvo, SP, onde foi conduzido o trabalho, apresenta $70 \%$ de seu território em remanescentes da Floresta Ombrófila Densa Atlântica (Rousselet-Gadenne, 2004).

A região apresenta relevo acidentado, com altitudes até $1.000 \mathrm{~m}$. O clima é Subtropical Úmido Mesotérmico (Cfb nas porções mais elevadas e Cfa nas mais baixas, conforme a classificação climática de Köppen), com estação quente e úmida de setembro a março, precipitação média anual variando de $1.500 \mathrm{~mm}$ a $2.000 \mathrm{~mm}$, e temperatura média anual de $21,5{ }^{\circ} \mathrm{C}$ (Rousselet-Gadenne, 2004; Instituto Paranaense de Desenvolvimento Econômico e Social, 2007). Na região destacam-se neossolos, argissolos, cambissolos e afloramentos rochosos (Sociedade de Pesquisa em Vida Selvagem , 1996; Embrapa Solos, 2006).

O trabalho foi conduzido em propriedades rurais contendo SAF multiestrata (AF) - composto por espécies de interesse agrícola (sobretudo a banana), cultivadas concomitantemente a espécies florestais de usos múltiplos, incluindo nativas e exóticas e manejadas de modo agroecológico, sem aportes externos de nutrientes e com mão de obra familiar e/ ou em sistema de mutirão - e em áreas agricultura convencional (AC), pastagem (PT) e capoeira (CP). Entre as áreas de agricultura estão incluídos plantios puros de abóbora (EA), de maracujá (NM) e de pupunha (NP) (para a produção de palmito), com a aplicação de adubação orgânica (composto elaborado a partir de cama de aviário) e mineral, e aplicação de defensivos químicos, enquanto as de pastagem incluem pastagens em diferentes graus de degradação (pouco ED, medianamente - LP, ou muito degradada - KK). Para os SAFs e as capoeiras, foram demarcadas áreas com diferentes idades para avaliar, também, o grau de desenvolvimento desses sistemas. Nos SAFs foram utilizadas idades de 4 (CL), 8 (SD) e 16 (SZ) anos e, nas capoeiras, 5 (C5), 20 (C20) e 30 (C30) anos. Ressaltamos 
também que as capoeiras com 5 e 20 anos de pousio já são averbadas como Reserva Legal (RL), enquanto que aquela de 30 anos compreende uma Área de Preservação Permanente (APP). As idades das capoeiras foram informadas pelos proprietários rurais e a escolha dessas áreas foi decorrente de sua situação jurídica, como APP ou RL, importante na comparação do SAF multiestrato com áreas de preservação e de conservação preconizadas no Código Florestal (BRASIL, 1965), objetivo principal do projeto no qual se insere o presente trabalho.

Para cada sistema de uso da terra (SUT) foram amostradas três propriedades rurais, nas quais foram instaladas parcelas de $25 \mathrm{~m} \times 10 \mathrm{~m}$. No total, cada SUT foi composto por nove parcelas, localizadas entre os paralelos $24^{\circ} 43^{\prime} 54^{\prime \prime} \mathrm{S}$ e $24^{\circ} 53^{\prime} 12^{\prime \prime} \mathrm{S}$, entre os meridianos $48^{\circ} 28^{\prime} 05^{\prime}$ ' W e $48^{\circ} 33^{\prime} 38^{\prime \prime} \mathrm{W}$, e entre 90 $\mathrm{m}$ e $223 \mathrm{~m}$ de altitude, numa área total de $49,8 \mathrm{~km}^{2}$. Nessas parcelas foram coletadas e analisadas as amostras de solo e de serapilheira, e realizados o inventário fitossociológico e a determinação do estoque de carbono em cada um dos compartimentos. A estimativa de estoque de carbono foi realizada segundo metodologia desenvolvida pelo International Centre for Research in Agroforestry (Icraf) (Arevalo et al., 2002), calculada pelo somatório do carbono estocado no solo e na biomassa vegetal definida, segundo esses autores, pelo somatório da serapilheira, da biomassa arbórea e da biomassa herbácea.

Foram coletadas três amostras dos horizontes superficiais (A) dos solos, nas profundidades de 0 a 10 $\mathrm{cm}$ e de $10 \mathrm{~cm}$ a $20 \mathrm{~cm}$, em cada um dos doze SUTs, totalizando 36 amostras de cada profundidade, sendo determinado o $\mathrm{pH}$, carbono e dos elementos químicos necessários para o cálculo da saturação em bases (V\%) e saturação em alumínio (m\%) (Claessen, 1997).

Para as análises físicas foram coletadas amostras indeformadas de solo utilizando-se cilindros metálicos de Kopec $\left(68,71 \mathrm{~cm}^{3}\right)$, nas mesmas profundidades, em cada um dos doze SUTs, totalizando nove amostras de cada profundidade. Para cada amostra foi determinada a densidade aparente (dap) e a porosidade volumétrica (pvol) (Claessen, 1997).

O carbono no solo (CS) foi determinado segundo as equações descritas por Arevalo et al. (2002):

(1) PVS $\left(\mathrm{Mg} \mathrm{ha}^{-1}\right)=$ dap x PS x 10.000, sendo PS (espessura do solo);

(2) $\mathrm{CS}\left(\mathrm{Mg} \mathrm{ha}^{-1}\right)=(\mathrm{PVS} \times \mathrm{CO}) / 100$.
Para a determinação do carbono estocado na serapilheira depositada sobre o solo, foram coletadas amostras em 27 parcelas $(50 \mathrm{~cm} \times 50 \mathrm{~cm})$ aleatoriamente demarcadas em cada SUT e que, após secas em estufa $\left(60^{\circ} \mathrm{C}\right)$ por até sete dias, foram separadas em (1) folhas; (2) galhos (com até $5 \mathrm{~cm}$ de diâmetro); (3) frutos (incluindo flores e sementes); (4) cascas e (5) raízes finas (presentes na camada de serapilheira depositada sobre o solo). Em seguida, as amostras foram moídas, peneiradas (em malha de $0,5 \mathrm{~mm}$ de diâmetro) sendo determinados os teores de carbono foram determinados pelo método de Walkley-Black, detalhado por Tedesco et al. (1995).

No inventário fitossociológico foram amostrados todos os indivíduos com DAP (diâmetro à altura do peito) igual ou superior a $5 \mathrm{~cm}$, os quais foram identificados e tiveram os valores de DAP e de altura total registrados. Para a vegetação herbáceo-arbustiva foram utilizadas 27 parcelas de $1 \mathrm{~m}^{2}$, por SUT, das quais foram retirados todos os indivíduos com até $1 \mathrm{~m}$ de altura, que foram identificados e pesados. O valor total de biomassa vegetal $\left(\mathrm{Mg} \mathrm{ha}^{-1}\right)$ foi obtido pela aplicação de equações alométricas descritas por Arevalo et al. (2002), para os indivíduos vivos $\left(B i v=0,1184 D A P^{2,53} \times 0,04\right)$, mortos $\left(B i m=0,1184 D A P^{2,53} \times 0,04\right)$, e aqueles da vegetação herbáceo-arbustiva $($ Breg $=(P S / P F) \times 0,04)$.

O cálculo do volume da biomassa aérea de todos os indivíduos, vivos e mortos (em pé), com DAP superior a $5 \mathrm{~cm}$ foi determinado mediante a utilização do programa Fitopac (Shepherd, 1995), e esses valores foram multiplicados por 0,45 para a estimativa do estoque de carbono na biomassa aérea, segundo Brown (1997).

A análise estatística foi realizada considerando que o experimento foi implantado em delineamento casualizado com três repetições, em arranjo fatorial hierárquico. Para garantir a análise de variância (ANOVA), foi realizado o teste de Kolmogorov-Smirnov para averiguar a normalidade dos dados. Quando detectadas distribuições não normais, foi realizada a análise dos dados por uma distribuição gama e análise de Deviance (Demétrio, 2002). Foram realizados os desmembramentos dos efeitos hierárquicos, e a comparação das médias dos diferentes tratamentos e propriedades foi realizada pelo Teste de Tukey, a 5\% de significância.

\section{Resultados e discussão}

A classe de solo predominante em quase todos os SUTs foi o Cambissolo Háplico típico A moderado 
(Tabela 1). Na propriedade cultivada com abóbora ocorreu o Cambissolo Flúvico situado em ambiente ribeirinho e cuja gênese sofre a influência de processos fluviais. Em uma das parcelas da propriedade cultivada com maracujá, registrou-se a presença de Neossolo Litólico. Tratam-se de solos pouco desenvolvidos, pouco profundos e profundos, com horizonte subsuperficial B incipiente (Cambissolos) ou com ausência do horizonte B diagnóstico (Neossolo). Na maioria dos casos, apresentam-se bem drenados e situam-se em relevo forte ondulado e ondulado. Os solos analisados apresentaram horizonte superficial moderado, com espessura entre $30 \mathrm{~cm}$ e $15 \mathrm{~cm}$, predominantemente, e textura média nas profundidades de $0-10 \mathrm{~cm}$ e $10-20 \mathrm{~cm}$ e argilosa no horizonte B (não coletado), com exceção apenas em dois SAFs com 5 anos, nos quais ocorreu horizonte A fraco, com espessura menor ou igual a $5 \mathrm{~cm}$.

$\mathrm{Na}$ Tabela 1 também constam as características químicas dos solos estudados. Quanto ao $\mathrm{pH}$, segundo Embrapa Solos (2006), a maioria dos solos apresentaramse fortemente e moderadamente ácidos ( $\mathrm{pH}$ entre 4,3 e 5,3 e entre 5,4 e 6,5 , respectivamente), a não ser os cultivados com abóbora, onde o $\mathrm{pH}$ foi praticamente neutro $(7,3)$.

Os valores mais baixos para CTC foram encontrados nos solos sob pastagem e capoeira com 5 anos. No primeiro caso, esta menor quantidade de carga, associada à menor quantidade de carbono e porosidade e maior densidade (Tabela 2), confirma o grau de degradação dos mesmos. No segundo, pode ser um indicativo do mau manejo, relativamente recente, aplicado a área antes da conversão para capoeira.

$\mathrm{Na}$ capoeira com 30 anos, em média, os solos apresentaram os menores valores de saturações em bases $(\mathrm{V} \%)$ e de $\mathrm{pH}$ e as maiores saturações em alumínio $(\mathrm{m} \%)$. O que pode ter ocorrido é que, neste grande lapso de tempo, houve um esgotamento das reservas de nutrientes possivelmente aplicados nas lavouras antecedentes. Entretanto, como será visto mais adiante, esta baixa fertilidade não impediu que o estoque de carbono total nas capoeiras fosse o mais elevado.

Após a observação visual de todas as áreas de cultivo e baseado na vivência dos próprios agricultores, esperavase que os solos sob os SAFs apresentassem melhorias na porosidade e na densidade em comparação à agricultura convencional e à pastagem, principalmente em função da densidade de raízes nos primeiros centímetros de solo e pela deposição da serapilheira e das estruturas vegetais decorrentes do manejo da poda, conforme observado na porosidade do solo sob capoeira com maior tempo de pousio $\left(\mathrm{C}_{30}\right.$, Pvol, Tabela 2$)$. Entretanto, a porosidade e a densidade dos solos sob SAF se assemelharam muito aos solos sob os sistemas agrícolas e as capoeiras, apenas diferindo significativamente das pastagens (Tabela 2, dados em negrito). Mesmo entre os diferentes SAFs, com tempo de maturação diferente, o SAF em formação, com apenas quatro anos de conversão do sistema tradicional de derrubada e queima para a agrofloresta (CL) apresentou-se menos compactado (menor densidade) que o do SAF com 16 anos de manejo.

As pastagens apresentaram maiores problemas quanto à compactação do solo, sobretudo nas propriedades com maior degradação do pasto (pastagem muito e medianamente degradada, respectivamente, ED e KK), nas quais o mesmo está mal formado, e com forte presença de diversas espécies espontâneas e de pteridófitas, geralmente indicadoras de acidez do solo. Na região estudada, mesmo na pastagem considerada pouco degradada (LP), composta uniformemente por Brachiaria spp., foi possível observar a forte presença de plantas daninhas e invasoras, tais como Cyperus meyenianus (tiririca) e alguns indivíduos de Psidium guajava (goiabeira), de porte arbóreo e arbustivo. 


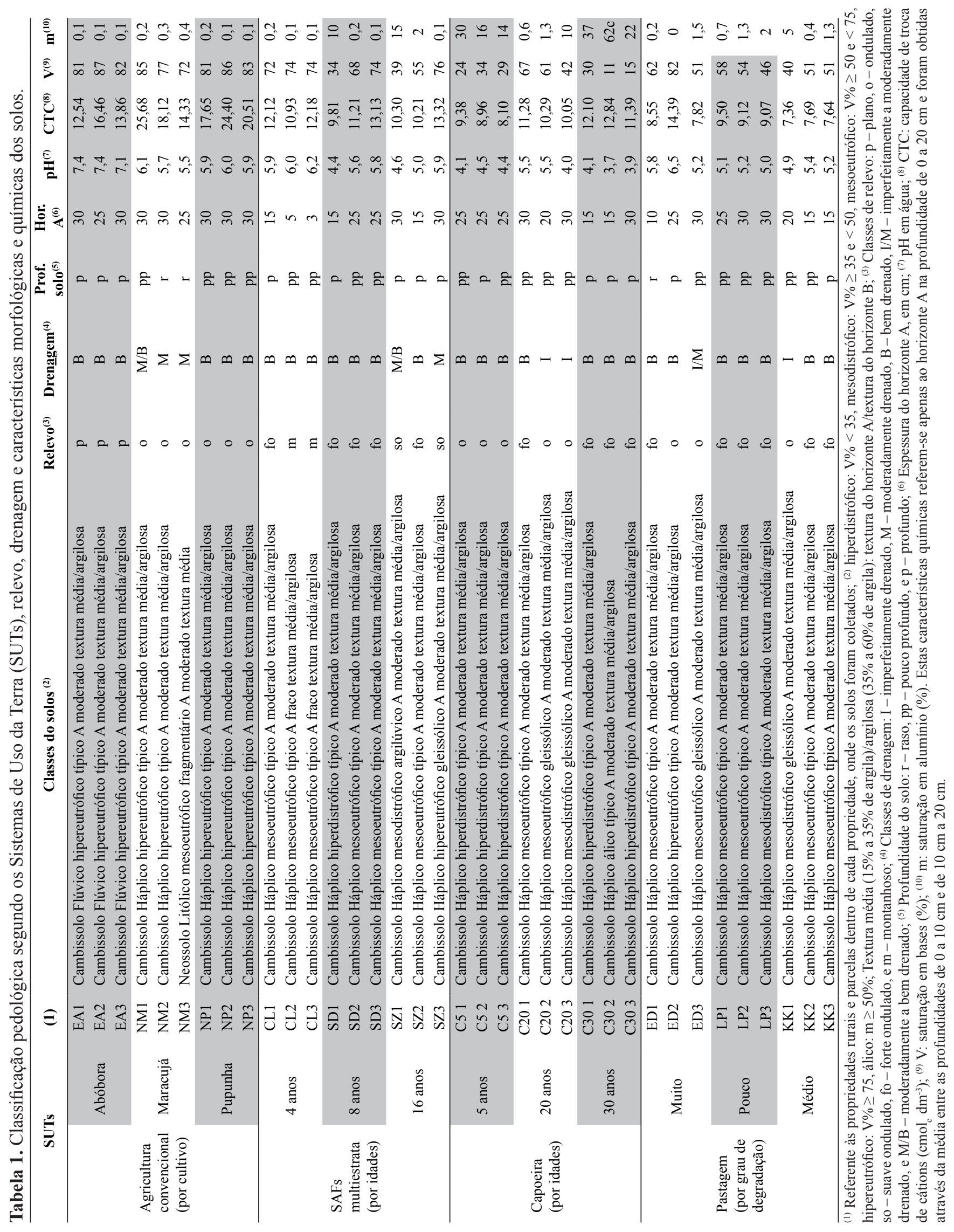


Tabela 2. Porosidade volumétrica (Pvol), densidade aparente (dap) e teor (CO) e estoque (CS) de carbono nos solos sob diferentes SUTs.

\begin{tabular}{|c|c|c|c|c|c|c|}
\hline \multicolumn{2}{|c|}{ SUTs } & Amostra & $\begin{array}{c}\text { Pvol } \\
\left(\mathrm{cm} \mathrm{cm}^{-3}\right)\end{array}$ & $\begin{array}{c}\text { Dap } \\
\left(\mathrm{kg} \mathrm{dm}^{-3}\right)\end{array}$ & $\begin{array}{c}\mathbf{C O} \\
\left(\mathrm{g} \mathrm{kg}^{-1}\right)\end{array}$ & $\begin{array}{c}\mathbf{C S} \\
\left(\mathrm{t} \mathrm{ha}^{-1}\right)\end{array}$ \\
\hline \multirow{4}{*}{$\begin{array}{l}\text { Agricultura } \\
\text { convencional } \\
\text { (por cultivo) }\end{array}$} & Abóbora & EA & $0,65 \mathrm{a}$ & $0,88 \mathrm{~b}$ & 20,08 a & $40,2 \mathrm{a}$ \\
\hline & Maracujá & NM & $0,56 \mathrm{~b}$ & $1,23 \mathrm{a}$ & $16,09 \mathrm{a}$ & $40,1 \mathrm{a}$ \\
\hline & Pupunha & NP & $0,55 \mathrm{~b}$ & $1,07 \mathrm{~b}$ & $21,46 \mathrm{a}$ & $34,4 \mathrm{a}$ \\
\hline & & & $0,58 \mathrm{~A}$ & $1,06 \mathrm{~B}$ & 19,21 A & $38,3 \mathrm{~A}$ \\
\hline \multirow{4}{*}{$\begin{array}{l}\text { SAFs } \\
\text { multiestrata } \\
\text { (por idades) }\end{array}$} & 4 anos & $\mathrm{CL}$ & $0,58 \mathrm{a}$ & $0,90 \mathrm{~b}$ & $19,94 \mathrm{a}$ & $29,9 \mathrm{~b}$ \\
\hline & 8 anos & SD & $0,55 \mathrm{a}$ & $1,06 \mathrm{ab}$ & $16,22 \mathrm{a}$ & $44,9 \mathrm{a}$ \\
\hline & 16 anos & $\mathrm{SZ}$ & $0,54 \mathrm{a}$ & $1,20 \mathrm{a}$ & $18,55 \mathrm{a}$ & $40,9 \mathrm{a}$ \\
\hline & & & 0,56 AB & $1,05 \mathrm{~B}$ & $18,23 \mathrm{AB}$ & 38,6 A \\
\hline \multirow{4}{*}{$\begin{array}{l}\text { Capoeira } \\
\text { (por idades) }\end{array}$} & 5 anos & $\mathrm{C} 5$ & $0,50 \mathrm{~b}$ & $1,07 \mathrm{a}$ & $16,23 \mathrm{~b}$ & $45,3 \mathrm{a}$ \\
\hline & 20 anos & $\mathrm{C} 20$ & $0,49 \mathrm{~b}$ & $0,97 \mathrm{a}$ & $19,50 \mathrm{a}$ & $34,4 \mathrm{a}$ \\
\hline & 30 anos & $\mathrm{C} 30$ & $0,57 \mathrm{a}$ & $0,99 \mathrm{a}$ & $19,73 \mathrm{a}$ & 37,3 a \\
\hline & & & 0,52 BC & 1,01B & 18,49 AB & 39,0 A \\
\hline \multirow{4}{*}{$\begin{array}{l}\text { Pastagem } \\
\text { (por grau de } \\
\text { degradação) }\end{array}$} & Muito & ED & $0,48 \mathrm{a}$ & $1,22 \mathrm{a}$ & $16,59 \mathrm{a}$ & $27,6 \mathrm{~b}$ \\
\hline & Pouco & LP & $0,51 \mathrm{a}$ & $1,10 \mathrm{~b}$ & $15,91 \mathrm{a}$ & $41,9 \mathrm{a}$ \\
\hline & Médio & KK & $0,47 \mathrm{a}$ & $1,30 \mathrm{a}$ & $14,39 \mathrm{a}$ & $32,3 \mathrm{~b}$ \\
\hline & & & $0,49 \mathrm{C}$ & $1,21 \mathrm{~A}$ & $15,63 \mathrm{~B}$ & 33,9 A \\
\hline
\end{tabular}

Em negrito, resultados médios encontrados para cada SUT avaliado. Em cada coluna, valores seguidos de letras minúsculas iguais não diferem significativamente entre si pelo Teste de Tukey a $5 \%$ de significância, dentro de cada SUT; valores seguidos de letras maiúsculas iguais não diferem significativamente entre os SUTs.

Em relação aos teores de carbono orgânico no solo (CO), os SAFs não diferiram dos demais sistemas de uso da terra (SUT) e também não houve diferença entre os SAFs em função do grau de maturação do sistema (Tabela 2), ao contrário do que foi observado entre as capoeiras. Maiores diferenças nos valores de $\mathrm{CO}$ só foram observados entre os sistemas agrícolas e as pastagens, provavelmente em função da adubação orgânica efetuada, sobretudo, nos plantios de maracujá e de pupunha, e pelo grau de degradação das pastagens, mesmo naquela menos degradada,.

Não foi constatada diferença significativa no estoque de carbono no solo entre os diferentes SUTs (Tabelas 2 e 3), com valores variando de 33,9 na pastagem a 39,0 $\mathrm{t} \mathrm{ha}^{-1} \mathrm{C}$ na capoeira.

A quantidade de carbono estocada na serapilheira variou de 1,61 a 5,95 $\mathrm{Mg} \mathrm{ha}^{-1}$, sendo que os estoques nas capoeiras e nos SAFs foram significativamente superiores aos dos sistemas agrícolas e das pastagens (Tabela 4, em negrito). Em todos os SUTs e em suas propriedades, os maiores estoques de carbono foram observados nas folhas da serapilheira, que representam de $48,7 \%$ a $85,8 \%$ do total da serapilheira depositada (capoeira e pastagem, respectivamente).
Diversos autores têm relatado a importância das folhas como a fração mais abundante na serapilheira depositada sobre o solo (Lodihiyal \& Lodihiyal, 1997; Herbohn \& Congdon, 1998; Laclau et al., 2003). Elas também constituem a fração predominante nos estudos de decomposição da serapilheira, e delas depende o estoque de nutrientes, a formação de uma manta orgânica sobre o solo e a sustentabilidade do sistema (Balieiro et al., 2003, 2004). Pela quantidade irregular de galhos, frutos, cascas e raízes, e sua sazonalidade, não foi observada diferença significativa para nenhuma dessas frações.

A partir das análises fitossociológicas, maiores estoques de carbono nas plantas herbáceo-arbustivas foram determinados nas pastagens $\left(0,012 \mathrm{Mg} \mathrm{ha}^{-1}\right)$, significativamente superior aos estoques nos sistemas agrícolas e nas capoeiras (ambas com $\left.0,005 \mathrm{Mg} \mathrm{ha}^{-1}\right)$. SAFs não diferiram dos demais sistemas e estocaram $0,011 \mathrm{Mg} \mathrm{ha}^{-1}$ de carbono (Tabela 3). O carbono estocado na biomassa aérea da capoeira $\left(70,82 \mathrm{Mg} \mathrm{ha}^{-1}\right)$ foi significativamente superior ao estocado nos SAFs $(32,36 \mathrm{Mg}$ $\mathrm{ha}^{-1}$ ), por sua vez, significativamente superior ao estocado nos sistemas agrícolas e nas pastagens $\left(7,20\right.$ e $0,30 \mathrm{Mg} \mathrm{ha}^{-1}$, respectivamente). 
Tabela 3. Estoque total de C e por compartimentos nos diferentes SUTs em $\mathrm{Mg} \mathrm{ha}^{-1}$.

\begin{tabular}{|c|c|c|c|c|c|c|c|c|}
\hline \multicolumn{2}{|c|}{ SUTs } & \multirow{2}{*}{$\begin{array}{c}\text { Propriedade } \\
\text { EA }\end{array}$} & \multirow{2}{*}{$\begin{array}{c}\begin{array}{c}\text { Volume } \\
\left(\mathbf{m}^{3}\right)\end{array} \\
0,00 \mathrm{a}\end{array}$} & \multirow{2}{*}{$\begin{array}{c}\begin{array}{c}\text { Biomassa } \\
\text { aérea } \\
\left(\mathbf{M g ~ h a}^{-1}\right)\end{array} \\
\text { nd }\end{array}$} & \multirow{2}{*}{$\begin{array}{c}\begin{array}{c}\text { Vegetação } \mathbf{h} \\
\text { erbáceo-arbustiva } \\
\left(\mathbf{M g ~ h a} \mathbf{~ h}^{-1}\right)\end{array} \\
0,006 \mathrm{a}\end{array}$} & \multirow{2}{*}{$\begin{array}{c}\begin{array}{c}\text { Serapilheira } \\
\left.\mathbf{( M g ~ h a}^{-1}\right)\end{array} \\
1,08 \mathrm{~b}\end{array}$} & \multirow{2}{*}{$\begin{array}{c}\begin{array}{c}\text { Solo } \\
\left(\mathbf{M g ~ h a}^{-1}\right)\end{array} \\
40,18 \mathrm{a}\end{array}$} & \multirow{2}{*}{$\begin{array}{c}\begin{array}{c}\text { Estoque } \\
\text { total } \\
\left(\mathbf{M g ~ h a}^{-1}\right)\end{array} \\
41,27 \mathrm{~b}\end{array}$} \\
\hline \multirow{4}{*}{$\begin{array}{l}\text { Agricultura } \\
\text { convencional } \\
\text { (por cultivo) }\end{array}$} & Abóbora & & & & & & & \\
\hline & Maracujá & NM & $0,00 \mathrm{a}$ & nd & $0,004 \mathrm{a}$ & $0,28 \mathrm{c}$ & $40,13 \mathrm{a}$ & $40,41 \mathrm{~b}$ \\
\hline & Pupunha & $\mathrm{NP}$ & $0,04 \mathrm{a}$ & 21,59 & $0,005 \mathrm{a}$ & $3,48 \mathrm{a}$ & $34,44 \mathrm{~b}$ & 59,52 a \\
\hline & & & 0,01 C & $7,20 \mathrm{C}$ & 0,005 B & 1,61 B & $38,25 \mathrm{~A}$ & $47,07 \mathrm{C}$ \\
\hline \multirow{4}{*}{$\begin{array}{l}\text { SAFs } \\
\text { multiestrata } \\
\text { (por idades) }\end{array}$} & 4 anos & $\mathrm{CL}$ & $2,04 \mathrm{a}$ & $31,83 \mathrm{a}$ & $0,004 \mathrm{~b}$ & $4,39 a b$ & $29,93 \mathrm{~b}$ & $66,15 \mathrm{~b}$ \\
\hline & 8 anos & SD & $1,96 \mathrm{a}$ & $35,47 \mathrm{a}$ & $0,016 \mathrm{a}$ & $4,19 \mathrm{~b}$ & $44,90 \mathrm{a}$ & $84,58 \mathrm{a}$ \\
\hline & 16 anos & $\mathrm{SZ}$ & $2,78 \mathrm{a}$ & 29,77 a & $0,013 \mathrm{a}$ & $4,65 \mathrm{a}$ & $40,95 \mathrm{a}$ & $75,38 a b$ \\
\hline & & & $2,26 \mathrm{~B}$ & 32,36 B & 0,011 AB & $4,41 \mathrm{~A}$ & 38,59 A & 75,37 B \\
\hline \multirow{4}{*}{$\begin{array}{l}\text { Capoeira } \\
\text { (por idades) }\end{array}$} & 5 anos & $\mathrm{C} 5$ & $4,64 \mathrm{~b}$ & $56,51 \mathrm{~b}$ & $0,007 \mathrm{a}$ & $5,31 \mathrm{~b}$ & $45,33 \mathrm{a}$ & $107,16 \mathrm{~b}$ \\
\hline & 20 anos & $\mathrm{C} 20$ & $5,62 \mathrm{~b}$ & $48,30 \mathrm{~b}$ & $0,003 \mathrm{a}$ & $7,25 \mathrm{a}$ & $34,42 \mathrm{~b}$ & $89,97 \mathrm{c}$ \\
\hline & 30 anos & C30 & $11,08 \mathrm{a}$ & 107,65 a & $0,005 \mathrm{a}$ & $5,27 \mathrm{~b}$ & $37,28 \mathrm{~b}$ & $150,21 \mathrm{a}$ \\
\hline & & & 7,11 A & $70,82 \mathrm{~A}$ & 0,005 B & 5,94 A & 39,01 A & $115,78 \mathrm{~A}$ \\
\hline \multirow{4}{*}{$\begin{array}{l}\text { Pastagem } \\
\text { (por grau de } \\
\text { degradação) }\end{array}$} & Muito & ED & $0,00 \mathrm{a}$ & nd & $0,014 \mathrm{a}$ & $3,25 \mathrm{a}$ & $27,59 \mathrm{c}$ & $30,85 \mathrm{~b}$ \\
\hline & Pouco & LP & $0,00 \mathrm{a}$ & nd & $0,010 \mathrm{a}$ & $0,69 \mathrm{c}$ & $41,89 \mathrm{a}$ & $42,59 \mathrm{a}$ \\
\hline & Médio & KK & $0,00 \mathrm{a}$ & nd & $0,011 \mathrm{a}$ & $2,29 \mathrm{~b}$ & $32,29 \mathrm{~b}$ & $34,59 \mathrm{ab}$ \\
\hline & & & $\mathbf{0 , 0 0} \mathrm{C}$ & nd & 0,012 A & $2,08 \mathrm{~B}$ & 33,92 A & $36,01 \mathrm{C}$ \\
\hline
\end{tabular}

Em negrito, resultados encontrados para cada sistema avaliado. Em cada coluna, valores seguidos de letras minúsculas iguais não diferem significativamente entre si pelo Teste de Tukey a 5\% de significância, comparando-se os "cultivos" dentro de cada SUT; valores seguidos de letras maiúsculas iguais não diferem significativamente entre os SUTs.

Tabela 4. Estoque de carbono $\left(\mathrm{Mg} \mathrm{ha}^{-1}\right)$ estocado na serapilheira (folhas, galhos, frutos, cascas, raízes) sobre os solos nas propriedades amostradas dentro de cada SUT (agricultura convencional, SAF, capoeira e pastagem).

\begin{tabular}{|c|c|c|c|c|c|c|c|}
\hline \multirow{2}{*}{\multicolumn{2}{|c|}{ SUTs }} & Folhas & Galhos & Frutos & Cascas & Raízes & Total \\
\hline & & \multicolumn{6}{|c|}{$\mathrm{C}\left(\mathrm{Mg} \mathrm{ha}^{-1}\right)$} \\
\hline \multirow{4}{*}{$\begin{array}{l}\text { Agricultura } \\
\text { convencional } \\
\text { (por cultivo) }\end{array}$} & Abóbora & $0,698 \mathrm{~b}$ & 0,485 a & nd & $0,008 \mathrm{a}$ & $0,349 \mathrm{a}$ & $1,082 \mathrm{~b}$ \\
\hline & Maracujá & $0,206 \mathrm{~b}$ & $0,106 \mathrm{a}$ & $0,011 \mathrm{a}$ & $\mathrm{Nd}$ & $0,042 \mathrm{a}$ & $0,257 \mathrm{~b}$ \\
\hline & Pupunha & $2,971 \mathrm{a}$ & $0,577 \mathrm{a}$ & $0,069 \mathrm{a}$ & $0,260 \mathrm{a}$ & nd & $3,481 \mathrm{a}$ \\
\hline & & $1,292 \mathrm{C}$ & 0,389 A & 0,029 A & $0,090 \mathrm{~A}$ & 0,130 A & 1,607 B \\
\hline \multirow{4}{*}{$\begin{array}{l}\text { SAFs } \\
\text { multiestrata } \\
\text { (por idades) }\end{array}$} & 4 anos & $2,379 \mathrm{a}$ & $1978,5 \mathrm{a}$ & $0,182 \mathrm{a}$ & $0,042 \mathrm{a}$ & $0,027 \mathrm{a}$ & $4,392 \mathrm{a}$ \\
\hline & 8 anos & $2,352 \mathrm{a}$ & $1979,4 \mathrm{a}$ & $0,078 \mathrm{a}$ & $0,051 \mathrm{a}$ & $0,086 \mathrm{a}$ & 4,194 a \\
\hline & 16 anos & $2,676 \mathrm{a}$ & $1677,3 \mathrm{a}$ & $0,048 \mathrm{a}$ & $0,177 \mathrm{a}$ & $0,586 \mathrm{a}$ & $4,646 \mathrm{a}$ \\
\hline & & $2,465 \mathrm{AB}$ & $1,878 \mathrm{~A}$ & 0103 A & 0,091 A & 0,234 A & 4,411 A \\
\hline \multirow{4}{*}{$\begin{array}{l}\text { Capoeira } \\
\text { (por idades) }\end{array}$} & 5 anos & $2,889,9 \mathrm{ab}$ & $1466,6 \mathrm{a}$ & $0,066 \mathrm{a}$ & $0,154 \mathrm{a}$ & $0,891 \mathrm{a}$ & $5,307 \mathrm{a}$ \\
\hline & 20 anos & $4,017,2 \mathrm{a}$ & 2975,6 a & $0,322 \mathrm{a}$ & 0,144 a & $0,422 \mathrm{a}$ & $7,255 \mathrm{a}$ \\
\hline & 30 anos & $1,785,9 \mathrm{~b}$ & 2478,1 a & $0,315 \mathrm{a}$ & $0,143 \mathrm{a}$ & $0,669 \mathrm{a}$ & $5,274 \mathrm{a}$ \\
\hline & & $2,898 \mathrm{~A}$ & 2,307 A & 0,235 A & 0,148 A & 0,661 A & 5,945 A \\
\hline \multirow{4}{*}{$\begin{array}{l}\text { Pastagem } \\
\text { (por grau de } \\
\text { degradação) }\end{array}$} & Muito & $2,916,4 \mathrm{a}$ & $322,3 \mathrm{a}$ & nd & $\mathrm{Nd}$ & $0,015 \mathrm{a}$ & $3,250 \mathrm{a}$ \\
\hline & Pouco & $0,502,3 \mathrm{~b}$ & $207,6 \mathrm{a}$ & nd & 0,004 & $0,070 \mathrm{a}$ & $0,689 \mathrm{~b}$ \\
\hline & Médio & $1,928,5 \mathrm{a}$ & $202,3 \mathrm{a}$ & 0,331 & $\mathrm{Nd}$ & $0,193 \mathrm{a}$ & $2,289 \mathrm{ab}$ \\
\hline & & $1,782 \mathrm{BC}$ & 0,244 A & $0,110 \mathrm{~A}$ & 0,001 A & $0,093 \mathrm{~A}$ & $2,076 \mathrm{~B}$ \\
\hline
\end{tabular}

Em negrito, resultados encontrados para cada sistema avaliado. Em cada coluna, valores seguidos de letras minúsculas iguais não diferem significativamente entre si pelo Teste de Tukey a 5\% de significância, comparando-se os "cultivos" dentro de cada SUT; valores seguidos de letras maiúsculas iguais não diferem significativamente entre os SUTs. 
No compartimento solo não houve diferenças significativas quanto ao estoque de carbono entre os diferentes SUTs (Tabela 4), com valores variando de 33,9 a 39,0 tha $^{-1} \mathrm{C}$, evidenciando a baixa fertilidade natural desses solos, e a baixa espessura do horizonte A, independente da cultura e/ou SUT sobre eles. No cômputo geral de todas as fontes de estoque de carbono desses sistemas, maiores quantidades de carbono estocado ocorrem nas capoeiras, seguidas dos $\mathrm{SAFs}$ e, em conjunto, dos sistemas agrícolas e das pastagens (Tabela 4), obedecendo as mesmas tendências observadas para os estoques na biomassa arbórea, variando de 36,01 a 115,8 $\mathrm{Mg} \mathrm{ha}^{-1}$ de carbono estocado nos SUTs.

A quantidade de carbono estocada nos solos avaliados encontra-se dentro da faixa de valores listados por Sauerbeck (2001) e Britez et al. (2006). Powlson \& Jenkinson (1981) afirmam que diferentes sistemas de uso do solo não têm habilidade de modificar os estoques de carbono no solo, apenas os redistribuem em profundidade. Adicionalmente, Bayer et al. (2006) afirmam que mudanças nas quantidades de $\mathrm{C}$ estocado ocorreriam, unicamente, em função do manejo do solo, principalmente quanto ao controle dos processos erosivos. No presente trabalho, apenas três parcelas avaliadas (8,33\%) não apresentavam solos com alta susceptibilidade à erosão (Dantas et al., 2005).

Do ponto de vista do manejo dos solos dessa região, observa-se que o relevo acidentado constitui-se no fator limitante a todas as propriedades avaliadas, uniformizando o manejo empregado nesses solos. Entre todas as parcelas, somente nas ocupadas com o plantio de maracujá foi observado o uso de implementos agrícolas mecanizados, sendo que esta prática, associada à ausência de cobertura do solo, se refletiu na maior compactação destes solos. Além disso, essa área comporta faixas de neossolo litólico, sujeitos a intensas remoções de nutrientes pela alta susceptibilidade à erosão hídrica, e que, por suas características, são indicados apenas para a prática silvicultural (Dedecek et al., 2007), ou como áreas de preservação permanente (Santos \& Klamt, 2004; Barbosa, 2006).

As demais áreas, constituídas por Cambissolos Háplicos, com sua grande variação quanto à fertilidade, baixa capacidade de mecanização e alta susceptibilidade à erosão, são indicadas para utilização em lavouras de baixo nível tecnológico (exceto as situadas em relevo forte ondulado e montanhoso), para o manejo com pastagens plantadas, para a silvicultura, para a preservação permanente ou para o reflorestamento com espécies nativas (Reatto et al., 2004; Pedron et al., 2006). Assim, em todas as áreas analisadas, observou-se a aptidão predominantemente florestal dos solos. Adicionalmente, as áreas ocupadas com o plantio de abóbora, por estarem localizadas na faixa marginal do Rio Turvo, constittuem APPs e deveriam ter seu sobremanejo interrompido, para promover a sucessão natural, para recuperação da vegetação nativa (Giupponi et al., 2006).

As capoeiras e os SAFs apresentaram maior diversidade total de espécies que os sistemas agrícolas e as pastagens (Figura 1). Catovsky et al. (2002) afirmam que quanto maior a diversidade de espécies em um determinado ecossistema, maior será o potencial de estoque de carbono na biomassa, em função da otimização da fotossíntese das diferentes espécies que compõem o ecossistema. Desta forma, os SAFs multiestrata, por sua diversidade horizontal e vertical, tendem a proporcionar estoques de carbono similares a ambientes naturalmente biodiversos. Do ponto de vista conservativo, por sua complexidade, além de possuírem potencial de sequestro de carbono similar às áreas de capoeira, esses SAFs ainda apresentam-se como uma alternativa importante para aliar produção de alimento com baixos impactos sobre os recursos naturais.

Os SAFs multiestratificados, apesar de amplamente difundidos como prática agrícola alternativa em várias regiões tropicais, ainda carecem de muita investigação e/ou de experimentação para elucidar as lacunas de conhecimento técnico-científico sob o ponto de vista de suas interações, potencialidades e manejo. Há ainda uma bibliografia incipiente, inclusive em relação ao tema abordado no presente trabalho, já que muitos dos modelos de SAFs multiestratos implantados nasceram de experiências pessoais e/ou do resgate de conhecimentos de agricultores e foram fruto da necessidade de se produzir alimentos de maneira econômica, social ou ambientalmente mais sustentável, sem o rigor acadêmico ou científico, como o que ocorreu nas áreas avaliadas.

Em um desses raros trabalhos, Mutuo et al. (2005) afirmam que SAFs multiestratificados possuem a capacidade de recuperar os estoques de carbono em ecossistemas terrestres, em quantidades inferiores, porém com taxas similares a sistemas de sucessão secundária (capoeiras), e o manejo adequado desses SAFs permite o desenvolvimento de um processo crescente de estoque de carbono, pelo aumento da eficiência, em vários estratos, da fotossíntese, gerando ganhos de produtividade e diminuindo significativamente as emissões de $\mathrm{CO}_{2}$ e outros GEEs $\left(\mathrm{N}_{2} \mathrm{O}\right.$ e $\left.\mathrm{CH}_{4}\right)$, em comparação a outros sistemas de cultivo e a ecossistemas florestais. 


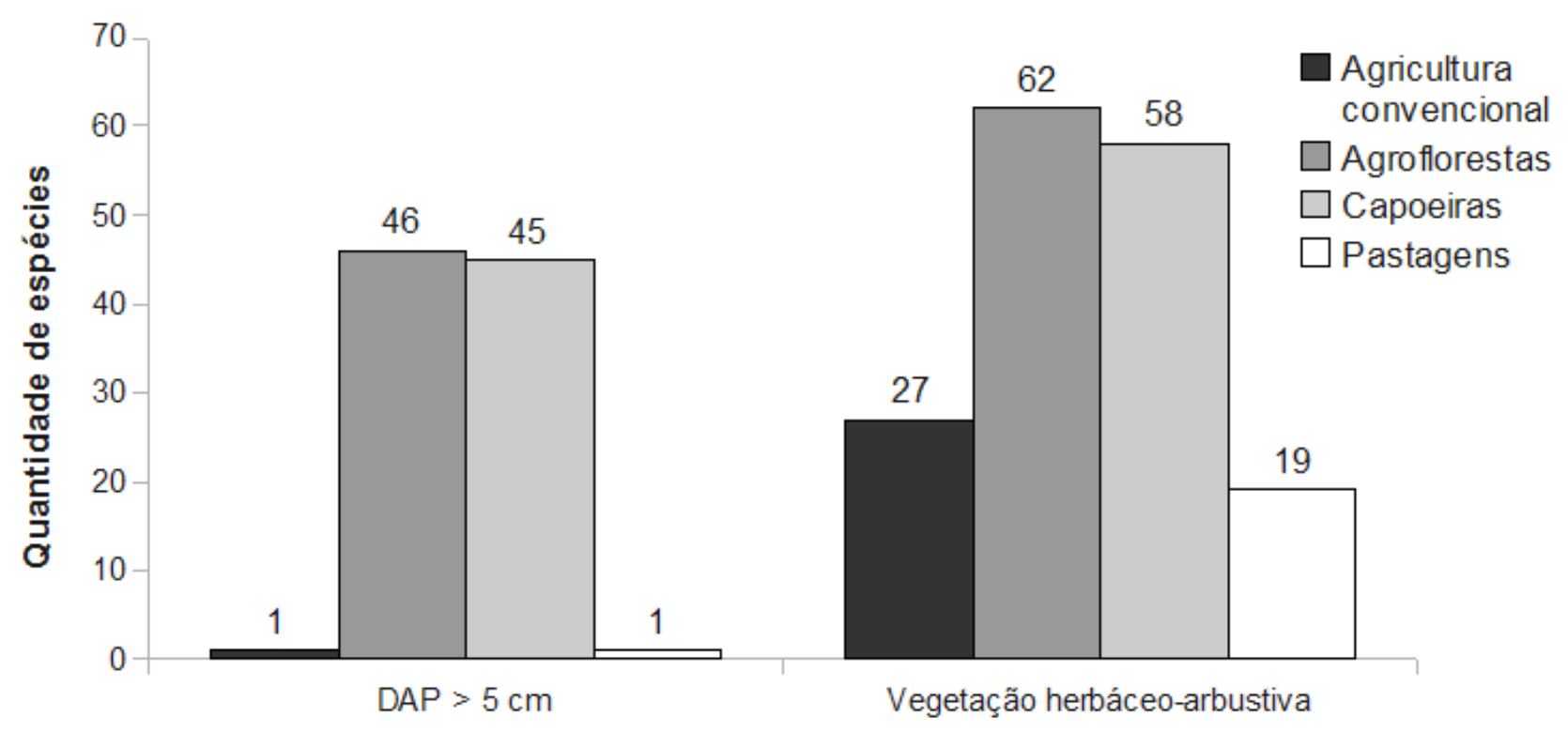

Figura 1. Diversidade de espécies encontradas nas áreas de agricultura convencional, agroflorestas, capoeiras e pastagens na região da Barra do Turvo, SP.

Apesar da falta de práticas silviculturais adequadas, as capoeiras (área natural, sem manejo) proporcionaram $119 \%$ a mais de carbono estocado na biomassa aérea do que nos SAFs (área manejada) avaliados. A alta densidade de plantas se, por um lado, pode ser positiva para a cobertura do solo, por outro, pode representar a estagnação desses SAFs como sistema produtivo de madeira e/ou de alimentos. Prova disso é a redução relativa dos valores de área basal e de volume total de biomassa entre o SAF mais novo (CL, 4 anos, 6,1 $\mathrm{m}^{3}$ ) e aquele mais velho (SZ, 16 anos, $8,4 \mathrm{~m}^{3}$ ), quando considerados a idade e o número de espécies dessas áreas. Igual comportamento foi observado por Baggio et al. (2009), nesses mesmos SAFs, sendo determinado que a baixa produção de madeira comercial foi decorrente da baixa tecnificação da produção e pela distribuição irregular de indivíduos entre os diferentes estratos arbóreos (predominância de indivíduos nos estratos superior e herbáceo, o que dificulta a sustentabilidade florestal).

Historicamente, os produtores agroflorestais associados à Cooperafloresta saíram de uma realidade, em que viviam abaixo da linha da pobreza, para dois salários mínimos mensais, em quinze anos de atividades. É compreensível, sob a ótica de sobrevivência dessas famílias que esses SAFs sejam continuadamente manejados para a produção agrícola, inclusive estendendo ao máximo os ciclos de produção das culturas agrícolas e/ou suprimindo o desenvolvimento das espécies florestais largamente plantadas. Um exemplo desse manejo florestal inadequado é a realização, por parte de alguns produtores, de podas constantes em indivíduos de espécies madeiráveis, visando à incorporação da biomassa aérea produzida ao solo ao invés de conduzilas para a produção florestal por meio de técnicas silviculturais apropriadas para produção de madeira para desdobro.

Contudo, apesar das capoeiras apresentarem maior acúmulo de biomassa que os SAFs, há que se considerar que a diferença de idade entre as áreas, bem como restrições legais ao uso florestal das espécies das capoeiras minimizam essas áreas como produtoras de produtos florestais madeiráveis à propriedade rural. Assim, os SAFs multiestrato avaliados apresentam-se como alternativas viáveis para a produção de alimentos e de produtos madeiráveis e não madeiráveis dentro da pequena e média propriedade rural, necessitando, contudo, experimentação complementar para definir, além do manejo mais correto em todas as suas fases de produção agroflorestal, qual é a melhor densidade desses sistemas para promover os ganhos ambientais e econômicos exigidos, dentre os quais o seu potencial como sequestrador de $\mathrm{CO}_{2}$, e sua elegibilidade em projetos de Mecanismo de Desenvolvimento Limpo 
(MDL), para promover os ganhos ambientais, florestais e econômicos pressupostos.

\section{Conclusões}

- Os sistemas de uso da terra (SUT) não representaram diferenças para o estoque de carbono no solo;

- Os SAFs multiestrata apresentam potencial similar de estoque de carbono na serapilheira quando comparados às áreas de capoeira, ambos superiores aos demais sistemas de uso da terra;

- As capoeiras apresentam maior estoque de carbono na biomassa aérea que os SAFs e ambos foram superiores aos demais SUTs. Igual comportamento foi observado para o total de carbono estocado;

- Apesar do grande potencial de sequestro de carbono dos SAFs, há necessidade de melhoria em suas práticas silviculturais.

\section{Agradecimentos}

Aos produtores rurais de Barra do Turvo, SP; ao técnico Benedito Jesus de Oliveira, da Coordenadoria de Assistência Técnica Integral, SP (Cati); ao produtor Claudinei Maciel dos Santos, então presidente da Associação dos Produtores Agroecológicos de Barra do Turvo e Adrianópolis (Cooperafloresta); aos técnicos florestais Roberto Carletto e Wilson Maschio, da Embrapa Florestas, e demais técnicos do Laboratório de Solos e Nutrição, e ao prof. Franklin Galvão (UFPR).

\section{Referências}

ANGEL-PÉREZ, A. L. D.; MENDOZA, B. M. A. Totonac homegardens and natural resources in Veracruz, Mexico. Agriculture and Human Values, v. 21, n. 4, p. 329-346, 2004.

AREVALO, L. A.; ALEGRE, J. C.; VILCAHUAMAN, L. J. M. Metodologia para estimar o estoque de carbono em diferentes sistemas de uso da terra. Colombo: Embrapa Florestas, 2002. (Embrapa Florestas. Documentos, 73).

BAGGIO, A. J. SOARES, A. O.; MASCHIO, W. O estrato arbóreo nos sistemas agroflorestais: um estudo de caso e perspectivas do mercado para espécies nativas. Colombo: Embrapa Florestas, 2009. 52 p.

BALIEIRO, F. C.; DIAS, L. E.; FRANCO, A. A.; CAMPELLO, E. F. C.; FARIA, S. M. Acúmulo de nutrientes na parte aérea, na serapilheira acumulada sobre o solo e decomposição de filódios de Acacia mangium Willd. Ciência Florestal, Santa Maria, v. 14, n. 1, p. 59-65, 2003.
BALIEIRO, F. C.; FRANCO, A. A.; PEREIRA, M. G.; CAMPELLO, E. F. C.; DIAS, L. E.; FARIA, S. M.; ALVES, B. J. R. Dinâmica da serapilheira e transferência de nitrogênio ao solo, em plantios de Pseudosamanea guachapele e Eucalyptus grandis. Pesquisa Agropecuária Brasileira, Brasília, DF, v. 39, n. 6, p. 597-601, 2004.

BARBOSA, L. M. (Coord). Manual para recuperação de áreas degradadas do Estado de São Paulo: matas ciliares do interior paulista. São Paulo: Instituto de Botânica, 2006. 129 p.

BAYER, C.; MARTIN-NETO, L.; MIELNICZUK, J.;

PAVINATO, A.; DIECKOW, J. Carbon sequestration in two Brazilian Cerrado soils under no-till. Soil \& Tillage Research, v. 86, p. 237-245, 2006.

BRASIL. Lei 4.771 de 15 de setembro de 1965. Institui o Novo Código Florestal . Disponível em: $<$ http://www.planalto.gov.br/ ccivil 03/Leis/L4771.htm>. Acesso em: 21 mar. 2010.

BRITEZ, R. M.; BORGO, M.; TIEPOLO, G.; FERRETTI, A.; CLAMON, A.; HIGA, R. Estoque e incremento de carbono em florestas e povoamentos de espécies arbóreas com ênfase na Floresta Atlântica do sul do Brasil. Colombo: Embrapa Florestas, 2006. 165 p.

BROWN, S. Estimating biomass and biomass changing of tropical forests: a primer. FAO Forestry Paper, Rome, v. 134, p. 1-55, 1997.

CAJA-GIRON, Y. S.; SINCLAIR, F. L. Characterization of multistrata silvopastoral systems on seasonally dry pastures in the Caribbean Region of Colombia. Agroforestry Systems, v. 53, p. 215-225, 2001

CATOVSKY, S.; BRADFORD, M. A.; HECTOR, A. Biodiversity and ecosystem productivity: implications for carbon storage. Oikos, v. 97, n. 3, p. 443-448, 2002.

CLAESSEN, M. E. C. (Org.). Manual de métodos de análise de solo. 2. ed. rev. atual. Rio de Janeiro: EMBRAPA-CNPS, 1997. 212 p. (EMBRAPA-CNPS. Documentos, 1).

DANTAS, M. E.; SHINZATO, E.; MEDINA, A. I. M.; SILVA, C. R.; PIMENTEL, J.; LUMBRERAS, J. F.; CALDERANO, S. B.; CARVALHO FILHO, A. Diagnóstico geoambiental do estado do Rio de Janeiro. In: OFICINA INTERNACIONAL DE ORDENAMENTO TERRITORIAL MINEIRO, CIENCIA Y TECNOLOGIA PARA EL DESAROLLO COOPERACION IBERO-AMERICANA,1., 2005, Rio de Janeiro. [Anais...] Rio de Janeiro: [s.n.], 2005. 1 CD-ROM. Sessão 4. 35 p. Disponível em: $<$ http://www.cprm.gov.br/publique/media/artigo_geoambientalRJ. pdf>. Acesso em: 1 abr. 2008.

DEDECEK, R. A.; CURCIO, G. R.; RACHWAL, M. F. G.; SIMON, A. A. Efeitos de sistemas de preparo do solo na erosão e na produtividade da acácia negra (Acacia mearnsii De Willd.). Ciência Florestal, v. 17, n. 3, p. 205-215, 2007

DEMÉTRIO, C. G. B. Modelos lineares generalizados em experimentação agronômica. Piracicaba, SP: ESALQ, 2002. 121 p. EMBRAPA SOLOS. Sistema brasileiro de classificação de solos. 2. ed. Rio de Janeiro, 2006. 306 p. 
FRANKE, I. L.; LUNZ, A. M. P.; AMARAL, E. F. Metodologia para planejamento, implantação e monitoramento de sistemas agroflorestais: um processo participativo. Rio Branco, AC: Embrapa Acre 1998. 3 p. (Embrapa Acre. Pesquisa em Andamento, 132)

GIUPPONI, C.; RAMANZIN, M.; STRARARO, E.; FUSER, S. Climate and land-use changes, biodiversity and agri-environmental measures in the Belluno province, Italy. Environmental Science and Policy, v. 9, p. 163-173, 2006.

GÖTSCH, E. Homem e natureza: cultura na agricultura. 2. ed. Recife: Centro de Desenvolvimento Agroecológico Sabiá, 1997. 17 p.

HERBOHN, J. L.; CONGDON, R. A. Ecosystem dynamics at disturbed and undisturbed sites in North Queensland wet tropical rain forest. III. Nutrient returns to the forest floor through litterfall. Journal of Tropical Ecology, v. 14, n. 2, p.217-229, 1998.

HOLGUÍN, V. A.; IBRAHIM, M.; MORA-DELGADO, J. El aprendizaje participativo como base de un cambio positivo del uso del suelo en fincas ganaderas de Costa Rica. Livestock

Research for Rural Development, v. 19, n. 4, abr. 2007. Disponível em: <http://www.cipav.org.co/lrrd/lrrd19/4/ holg19053.htm>. Acesso em: 28 jun. 2007.

ISAAC, M. E.; TIMMER, V. R.; QUASHIE-SAM, S. J. Shade tree effects in an 8-year-old cocoa agroforestry system: biomass and nutrient diagnosis of Theobroma cacao by vector analysis. Nutrient Cycling in Agroecosystems, v. 78, p. 155-165, 2007.

LACLAU, J.P.; DELEPORTE, P.; RANGER, J.; BOUILLET, J.P.; KAZOTTI, G. Nutrient dynamics throughout the rotation of Eucalyptus clonal stands in Congo. Annals of Botany, v. 91, p. 879-892, 2003.

LEHMANN, J.; GÜNTHER, D.; MOTA, M. S. da; ALMEIDA, M. P. de; ZECH, W.; KAISER, K. Inorganic and organic soil phosphorus and sulfur pools in an Amazonian multistrata agroforestry system. Agroforestry Systems, v. 53, n. 2, p. 113124, 2001.

LODHIYAL, L. S.; LODHIYAL, N. Nutrient cycling and nutrient use efficiency in short rotation, high density Central Himalayan Tarai Poplar Plantations. Annals of Botany, v. 79, p. 517-527, 1997.

MUTUO, P. K.; CADISCH, G.; ALBRECHT, A.; PALM, C. A.; VERCHOT, L. Potential of agroforestry for carbon sequestration and mitigation of greenhouse gas emissions from soils in the tropics. Nutrient Cycling in Agroecosystems, v. 71, p. 43-54, 2005.

NOBRE, C. A.; NOBRE, A. D. O balanço de carbono da Amazônia brasileira. Estudos Avançados, v. 16, n. 45, p. 81-90, 2002.

PEDRON, F. A.; POELKING, E. L.; DALMOLIN, R. S. D.; AZEVEDO, A. C.; KLAMT, E. A aptidão do uso da terra como base para o planejamento da utilização dos recursos naturais no Município de São João do Polêsine - RS. Ciência Rural, v. 36, n. 1, p. 105-112, 2006.

PENMAN, J.; KRUGER, D.; GALBALLY, I.; HIRAISHI, T.; NYENZI, B.; EMMANUEL, S.; BUENDIA, L.; HOPPAUS, R.; MARTINSEN, T.; MEIJER, J.; MIWA, K.; TANABE, K. Good practice guidance and uncertainty management in national greenhouse gas inventories: $\mathrm{N}_{2} \mathrm{O}$ emissions from adiphic acid and nitric acid production. Geneva: Intergovernmental Panel on Climate Change, 2000.
PENMAN, J.; KRUGER, D.; GALBALLY, I.; HIRAISHI, T.; NYENZI, B.; EMMANUEL, S.; BUENDIA, L.; HOPPAUS, R.; MARTINSEN, T.; MEIJER, J.; MIWA, K.; TANABE, K. Good practice guidance and uncertainty management in national greenhouse gas inventories: $\mathrm{N}_{2} \mathrm{O}$ emissions from adiphic acid and nitric acid production. Geneva: Intergovernmental Panel on Climate Change, 2000.

POWLSON, D. S.; JENKINSON, D. S. A comparison of the organic matter, biomass, adenosine triphosphate and mineralization nitrogen contents of ploughed and diect-drilled soils. Journal of Agricultural Science, v. 97, p. 713-721, 1981.

REATTO, A.; MARTINS, E. S.; FARIAS, M. F. R.; SILVA, A. V.; CARVALHO JUNIOR, O. A.; OLIVEIRA JUNIOR, R. C.; RODRIGUES, T. E.; SANTOS, P. L.; VALENTE, M. A. Mapa pedológico digital: SIG atualizado do Distrito Federal escala 1:100.000 e uma síntese do texto explicativo. Planaltina, DF: Embrapa Cerrados, 2004. 31 p. (Embrapa Cerrados. Documentos, 120).

RIBEIRO, M. C.; METZGER, J. P.; MARTENSEN, A. C.; PONZONI, F. J.; HIROTA, M. M. The Brazilian Atlantic Forest: how much is left, and how is the remaining forest distributed? Implications for conservation. Biological Conservation, v. 142, n. 6, p. 1141-1153, June 2009.

ROUSSELET-GADENNE, A. Adoption d'innovations agroforestières à Barra do Turvo (São Paulo, Brésil). Cahiers d'Agriculture, Paris, v. 13, n. 5, p. 391-402, 2004.

SANTOS, F. J.; KLAMT, E. Gestão agroecológica de microbacias hidrográficas através de técnicas de geoprocessamento e sensoriamento remoto: caso fazenda Pantanoso. Ciência Rural, v. 34, n. 6, p. 1785-1792, 2004.

SANTOS, M. J. C.; RODRIGUEZ, L. C. E.; WANDELI, E. V. Avaliação econômica de quatro modelos agroflorestais em áreas degradadas por pastagens na Amazônia Ocidental. Scientia Florestalis, Piracicaba, SP, v. 62, p. 48-61, 2002.

SAUERBECK, D. R. $\mathrm{CO}_{2}$ emissions and $\mathrm{C}$ sequestration by agriculture: perspectives and limitations. Nutrient Cycling in Agroecosystems, v. 60, p. 253-266, 2001.

SCHROTH, G.; D’ANGELO, S. A.; TEIXEIRA, W. G.; HAAG, D.; LIEBEREI, R. Conversion of secondary forest into agroforestry and monoculture plantations in Amazonia: consequences for biomass, litter and soil carbon stocks after 7 years. Forest Ecology and Management, v. 163, p. 131-150, 2002.

SHEPHERD, G. J. FITOPAC 1: Manual de usuário. Campinas, SP: Departamento de Botânica, UNICAMP, 1995. 93 p.

SOCIEDADE DE PESQUISA EM VIDA SELVAGEM. Manual para recuperação da reserva florestal legal. Curitiba: FNMA, 1996. $84 \mathrm{p}$.

STAVER, C.; GUHARAY, F.; MONTEROSSO, D.; MUSCHLER, R. G. Designing pest-supressive multiestrata perennial crop systems: shade-grown coffee in Central America. Agroforestry Systems, v. 53, p. 151-170, 2001.

SUYANTO, S.; PERMANA, R. P.; KHUSUSIYAH, N.; JOSHI, L. Land tenure, agroforestry adoption, and reduction of fire hazard in a forest zone: a case study from Lampung, Sumatra, Indonesia. Agroforestry Systems, v. 65, p. 1-11, 2005.

Pesq. flor. bras., Colombo, v. 31, n. 66, p. 143-154, abr./jun. 2011 
TEDESCO, M. J.; GIANELLO, C.; BISSANI, C. A.; BOHNEN. H.; VOLKWEISS, S. J. Análises de solo, plantas e outros materiais. Porto Alegre: Faculdade de Agronomia, UFRGS, 1995. 188 p. (Boletim Técnico de Solos, 5).

VELOSO, H. P.; GÓES-FILHO, L. Fitogeografia Brasileira: Classificação Fisionômico-Ecológica da Vegetação Neotropical. Salvador, BA: RADAMBRASIL, 1982.85 p.
YU, C. M. Sequestro florestal do carbono no Brasil: dimensões políticas, socioeconômicas e ecológicas. São Paulo: Annablume; IEB, 2004. $200 \mathrm{p}$. 